\title{
Complexation of $\mathrm{Np}(\mathrm{V})$ with the dicarboxylates
}

malonate and succinate: complex stoichiometry, thermodynamic data and structural information - Supplementary information

Martin M. Maiwald ${ }^{1,2}$, Katharina Müller ${ }^{3}$, Karsten Heim $^{3}$, Jörg Rothe ${ }^{2}$, Kathy Dardenne ${ }^{2}$, André Rossberg $^{3}$, Daniel Fröhlich ${ }^{1}$, Carsten Koke ${ }^{1}$, Michael Trumm ${ }^{2}$, Andrej Skerencak-Frech ${ }^{2}$, Petra J. Panak ${ }^{1,2}$

1) Ruprecht Karls Universität Heidelberg, Physikalisch-Chemisches Institut, Im Neuenheimer Feld 253, D-69120 Heidelberg, Germany

2) Karlsruher Institut für Technologie (KIT), Institut für Nukleare Entsorgung (INE), D-76344 Eggenstein-Leopoldshafen, Germany

*E-Mail:m.maiwald@pci.uni-heidelberg.de

\section{Supporting Information}

\section{Tables}

Table S1: Thermodynamic stability constants $\log \beta_{n}^{0}(\Theta)$ for the formation of $\left[\mathrm{NpO}_{2}(\mathrm{Mal})_{n}\right]^{1-2 n}(n=1,2)$ obtained from $\mathrm{NaClO}_{4}$ and $\mathrm{NaCl}$ media and their mean values as a function of temperature.

\begin{tabular}{|c|c|c|c|c|c|c|c|c|c|}
\hline & $\Theta\left[{ }^{\circ} \mathrm{C}\right]$ & 20 & 30 & 40 & 50 & 60 & 70 & 80 & 85 \\
\hline \multirow{4}{*}{$\begin{array}{l}\frac{\dot{\bar{\pi}}}{\sum_{0}^{\pi}} \\
\frac{0}{2}\end{array}$} & \multirow[t]{2}{*}{$\mathrm{NaClO}_{4}$} & 3.31 & 3.38 & 3.38 & 3.48 & 3.48 & 3.52 & 3.54 & 3.61 \\
\hline & & 0.06 & 0.06 & 0.08 & 0.08 & 0.06 & 0.10 & 0.06 & 0.08 \\
\hline & \multirow[t]{2}{*}{$\mathrm{NaCl}$} & 3.32 & 3.43 & 3.45 & 3.53 & 3.53 & 3.56 & 3.58 & 3.61 \\
\hline & & 0.06 & 0.07 & 0.08 & 0.08 & 0.08 & 0.08 & 0.09 & 0.07 \\
\hline
\end{tabular}




\begin{tabular}{|c|c|c|c|c|c|c|c|c|c|}
\hline \multirow[t]{2}{*}{ 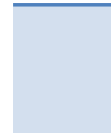 } & \multirow[t]{2}{*}{$\varnothing$} & 3.31 & 3.41 & 3.42 & 3.51 & 3.50 & 3.54 & 3.56 & 3.61 \\
\hline & & 0.07 & 0.08 & 0.09 & 0.08 & 0.09 & 0.08 & 0.09 & 0.08 \\
\hline \multirow{6}{*}{ 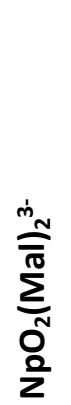 } & \multirow[t]{2}{*}{$\mathrm{NaClO}_{4}$} & 3.90 & 4.00 & 4.03 & 4.15 & 4.18 & 4.23 & 4.27 & 4.33 \\
\hline & & 0.06 & 0.08 & 0.08 & 0.06 & 0.06 & 0.10 & 0.08 & 0.12 \\
\hline & \multirow[t]{2}{*}{$\mathrm{NaCl}$} & 3.91 & 3.98 & 4.06 & 4.15 & 4.21 & 4.27 & 4.32 & 4.37 \\
\hline & & 0.11 & 0.07 & 0.10 & 0.09 & 0.14 & 0.10 & 0.10 & 0.10 \\
\hline & \multirow[t]{2}{*}{$\varnothing$} & 3.90 & 3.99 & 4.04 & 4.20 & 4.20 & 4.25 & 4.30 & 4.35 \\
\hline & & 0.11 & 0.08 & 0.10 & 0.09 & 0.14 & 0.11 & 0.11 & 0.12 \\
\hline
\end{tabular}

Table S2 Thermodynamic stability constants $\log \beta_{n}^{0}(\Theta)$ for the formation of $\left[\mathrm{NpO}_{2}(\mathrm{Succ})_{n}\right]^{1-2 n}(n=1,2)$ obtained from $\mathrm{NaClO}_{4}$ and $\mathrm{NaCl}$ media and their mean values as a function of temperature.

\begin{tabular}{|c|c|c|c|c|c|c|c|c|c|}
\hline & $\Theta\left[{ }^{\circ} \mathrm{C}\right]$ & 20 & 30 & 40 & 50 & 60 & 70 & 80 & 85 \\
\hline \multirow{6}{*}{ 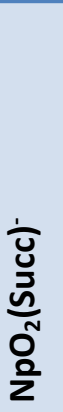 } & $\mathrm{NaClO}_{4}$ & 2.13 & 2.00 & 1.99 & 2.11 & 2.15 & 2.23 & 2.44 & 2.49 \\
\hline & & 0.12 & 0.09 & 0.07 & 0.06 & 0.06 & 0.09 & 0.17 & 0.12 \\
\hline & $\mathrm{NaCl}$ & 2.13 & 2.23 & 2.26 & 2.28 & 2.38 & 2.49 & 2.62 & 2.64 \\
\hline & & 0.10 & 0.10 & 0.08 & 0.08 & 0.11 & 0.06 & 0.11 & 0.18 \\
\hline & $\varnothing$ & 2.13 & 2.11 & 2.12 & 2.19 & 2.26 & 2.36 & 2.53 & 2.56 \\
\hline & & 0.15 & 0.14 & 0.10 & 0.10 & 0.12 & 0.11 & 0.20 & 0.22 \\
\hline \multirow{6}{*}{ 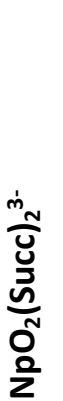 } & $\mathrm{NaClO}_{4}$ & & & & 1.32 & 1.51 & 1.61 & 1.80 & 2.13 \\
\hline & & - & - & - & 0.19 & 0.07 & 0.12 & 0.19 & 0.15 \\
\hline & $\mathrm{NaCl}$ & & & & 1.49 & 1.83 & 1.94 & 2.19 & 2.40 \\
\hline & & - & - & - & 0.10 & 0.14 & 0.11 & 0.11 & 0.19 \\
\hline & $\varnothing$ & & & & 1.41 & 1.67 & 1.77 & 2.00 & 2.26 \\
\hline & & - & - & - & 0.21 & 0.15 & 0.16 & 0.22 & 0.24 \\
\hline
\end{tabular}


Table S3: Fit parameters of the raw $\mathrm{k}^{2}$-weighted $\mathrm{Np}$ - $\mathrm{L}_{3}$-edge EXAFS spectra of $\mathrm{Np}(\mathrm{V})$ in the presence of malonate as a function of $\mathrm{pH}_{\mathrm{c}}$.

\begin{tabular}{|c|c|c|c|c|c|c|c|c|}
\hline \multicolumn{2}{|c|}{$\mathrm{pH}_{\mathrm{c}}$} & 7.8 & 5.2 & 5.0 & 4.7 & 4.4 & 3.8 & 3.1 \\
\hline \multirow{3}{*}{$\mathrm{O}_{\mathrm{ax}}$} & $\mathrm{N}$ & $2 *$ & $2 *$ & $2 *$ & 2* & 2* & $2 *$ & $2 *$ \\
\hline & $\mathrm{R} / \AA$ & $1.83(1)$ & $1.83(1)$ & $1.85(1)$ & $1.83(1)$ & $1.84(1)$ & $1.84(1)$ & $1.83(1)$ \\
\hline & $\sigma^{2} / \AA^{2}$ & $0.0002(3)$ & $0.0009(3)$ & $0.0006(4)$ & $0.0006(3)$ & $0.0004(2)$ & $0.0001(3)$ & $0.0017(2)$ \\
\hline \multirow{3}{*}{$\mathrm{O}_{\mathrm{mul}}$} & $\mathrm{N}$ & $4^{*}$ & $4^{*}$ & 4* & 4* & 4* & $4^{*}$ & $4^{*}$ \\
\hline & $\mathrm{R} / \AA$ & $3.67^{* *}$ & $3.66 * *$ & $3.70 * *$ & $3.66 * *$ & $3.68^{* *}$ & $3.67 * *$ & $3.66 * *$ \\
\hline & $\sigma^{2} / \AA^{2}$ & $0.0004 * *$ & $0.0019 * *$ & $0.0013^{* *}$ & $0.0012 * *$ & $0.0009 * *$ & $0.0001 * *$ & $0.0036 * *$ \\
\hline \multirow{3}{*}{$\mathrm{O}_{\mathrm{eq}}$} & $\mathrm{N}$ & $3.9(1.0)$ & $3.7(1.0)$ & $4.0(1.0)$ & $3.5(1.0)$ & $3.8(1.0)$ & $4.0(1.0)$ & $3.9(1.0)$ \\
\hline & $\mathrm{R} / \AA$ & $2.46(1)$ & $2.45(1)$ & $2.47(1)$ & $2.46(1)$ & $2.46(1)$ & $2.47(1)$ & $2.44(1)$ \\
\hline & $\sigma^{2} / \AA^{2}$ & $0.0062(14)$ & $0.0046(11)$ & $0.0070(20)$ & $0.0042(13)$ & $0.0066(12)$ & $0.0062(15)$ & $0.0096(16)$ \\
\hline \multirow{3}{*}{$\mathrm{C}_{\mathrm{c}}$} & $\mathrm{N}$ & $2.7(1.0)$ & $3.7(1.0)$ & $1.9(1.0)$ & $3.0(1.0)$ & $2.0(1.0)$ & $2.5(1.0)$ & $2.0(1.0)$ \\
\hline & $\mathrm{R} / \AA$ & $3.39(3)$ & $3.42(2)$ & $3.48(5)$ & $3.41(3)$ & $3.38(5)$ & $3.41(2)$ & $3.38(2)$ \\
\hline & $\sigma^{2} / \AA^{2}$ & $0.004^{*}$ & $0.004 *$ & $0.004 *$ & $0.004 *$ & $0.004 *$ & $0.004 *$ & $0.004^{*}$ \\
\hline \multicolumn{2}{|c|}{$\Delta \mathrm{E}_{\mathrm{o}} / \mathrm{eV}$} & $1.9(5)$ & $2.9(5)$ & $2.7(7)$ & $3.3(5)$ & $2.2(4)$ & $2.9(5)$ & $2.3(4)$ \\
\hline \multicolumn{2}{|c|}{ Red. error } & 0.014376 & 0.0157143 & 0.024140 & 0.0189825 & 0.0100117 & 0.0179982 & 0.00741869 \\
\hline
\end{tabular}

* Parameter fixed; ** parameter depending on $\mathrm{X}\left(\mathrm{O}_{\mathrm{ax}}\right): \mathrm{X}\left(\mathrm{O}_{\mathrm{mul}}\right)=2 \cdot \mathrm{X}\left(\mathrm{O}_{\mathrm{ax}}\right) \cdot \mathrm{O}_{\mathrm{ax}}=$ axial O-atoms, $\mathrm{O}_{\text {mul }}=$ multi scattering path of axial O-atoms $(\mathrm{Np}-\mathrm{O}-\mathrm{O}), \mathrm{O}_{\mathrm{eq}}=$ equatorial $\mathrm{O}$-atoms, $\mathrm{C}_{\mathrm{c}}=\mathrm{C}$-atoms of coordinating COO- groups. 
Table S4: Fit parameters of the raw $\mathrm{k}^{2}$-weighted $\mathrm{Np}$ - $\mathrm{L}_{3}$-edge EXAFS spectra of $\mathrm{Np}(\mathrm{V})$ in the presence of succinate as a function of $\mathrm{pH}_{\mathrm{c}}$.

\begin{tabular}{|c|c|c|c|c|c|c|}
\hline \multicolumn{2}{|c|}{$\mathrm{pH}_{\mathrm{c}}$} & 7.8 & 6.1 & 5.2 & 4.0 & 0.8 \\
\hline \multirow{3}{*}{$\mathrm{O}_{\mathrm{ax}}$} & $\mathrm{N}$ & $2^{*}$ & $2^{*}$ & $2^{*}$ & $2^{*}$ & $2^{*}$ \\
\cline { 2 - 7 } & $\mathrm{R} / \AA$ & $1.84(1)$ & $1.84(1)$ & $1.84(1)$ & $1.84(1)$ & $1.82(1)$ \\
\cline { 2 - 7 } & $\sigma^{2} / \AA^{2}$ & $0.0005(4)$ & $0.0001(4)$ & $0.0003(4)$ & $0.0014(4)$ & $0.0002(3)$ \\
\hline \multirow{3}{*}{$\mathrm{O}_{\text {mul }}$} & $\mathrm{N}$ & $4^{*}$ & $4^{*}$ & $4^{*}$ & $2^{*}$ & $4^{*}$ \\
\cline { 2 - 7 } & $\mathrm{R} / \AA$ & $3.46(3)$ & $3.46(3)$ & $3.50(3)$ & $3.58(3)$ & $3.56(3)$ \\
\cline { 2 - 7 } & $\sigma^{2} / \AA^{2}$ & 0.00100 & 0.0002 & 0.0005 & 0.0030 & 0.0005 \\
\hline \multirow{3}{*}{$\mathrm{O}_{\text {eq }}$} & $\mathrm{N}$ & $4.7(1.0)$ & $4.6(1.0)$ & $5.1(1.0)$ & $4.5(1.0)$ & $5.1(1.0)$ \\
\cline { 2 - 7 } & $\mathrm{R} / \AA$ & $2.49(1)$ & $2.48(1)$ & $2.49(1)$ & $2.50(1)$ & $2.49(1)$ \\
\cline { 2 - 7 } & $\sigma^{2} / \AA^{2}$ & $0.0062(18)$ & $0.0052(17)$ & $0.0065(9)$ & $0.0047(15)$ & $0.0079(21)$ \\
\hline \multirow{3}{*}{$\mathrm{C}_{\mathrm{c}}$} & $\mathrm{N}$ & $1.1(1.0)$ & $1.0(1.0)$ & $0.8(1.0)$ & $0.5(1.0)$ & - \\
\cline { 2 - 7 } & $\mathrm{R} / \AA$ & $2.88(4)$ & $2.78(5)$ & $2.84(6)$ & $2.81(1)$ & - \\
\cline { 2 - 7 } & $\sigma^{2} / \AA^{2}$ & $0.003^{*}$ & $0.003^{*}$ & $0.003^{*}$ & $0.003^{*}$ & - \\
\hline \multicolumn{2}{|c|}{$\Delta \mathrm{E}_{\mathrm{o}} / \mathrm{eV}$} & $7.9(6)$ & $7.5(5)$ & $8.0(6)$ & $8.6(5)$ & $7.2(6)$ \\
\hline \multicolumn{2}{|c|}{ Red. error } & 0.0164131 & 0.0185585 & 0.0242427 & 0.0221710 & 0.0201802 \\
\hline
\end{tabular}

* Parameter fixed; ** parameter depending on $\mathrm{X}\left(\mathrm{O}_{\mathrm{ax}}\right): \mathrm{X}\left(\mathrm{O}_{\text {mul }}\right)=2 \cdot \mathrm{X}\left(\mathrm{O}_{\mathrm{ax}}\right) \cdot \mathrm{O}_{\mathrm{ax}}=$ axial $\mathrm{O}$-atoms, $\mathrm{O}_{\text {mul }}=$ multi scattering path of axial O-atoms (Np-O-O), $\mathrm{O}_{\text {eq }}=$ equatorial O-atoms, $\mathrm{C}_{\mathrm{c}}=\mathrm{C}$-atoms of coordinating $\mathrm{COO}^{-}$groups.

\section{Figures}
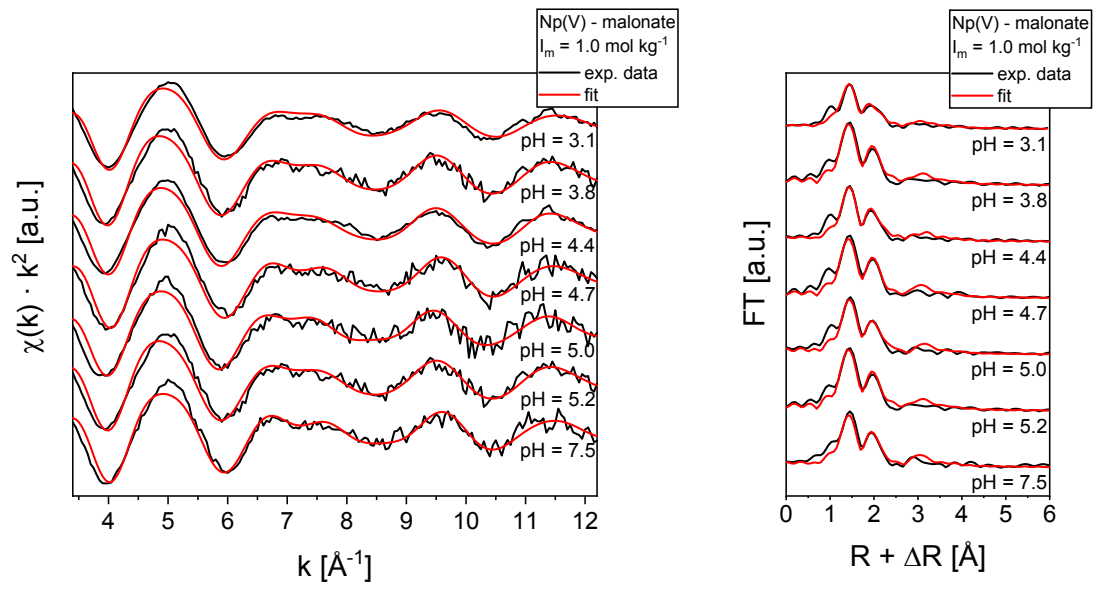

Figure S1: EXAFS analyses of $\mathrm{Np}(\mathrm{V})$ in the presence of malonate as a function of the conditional $\mathrm{pH}_{\mathrm{c}}$ value. (left) raw $\mathrm{k}^{2}$ weighted Np-L $\mathrm{L}_{3}$-edge EXAFS spectra; (right) related Fourier transforms; (black) experimental data; (red) best fit from EXAFSPAK. 

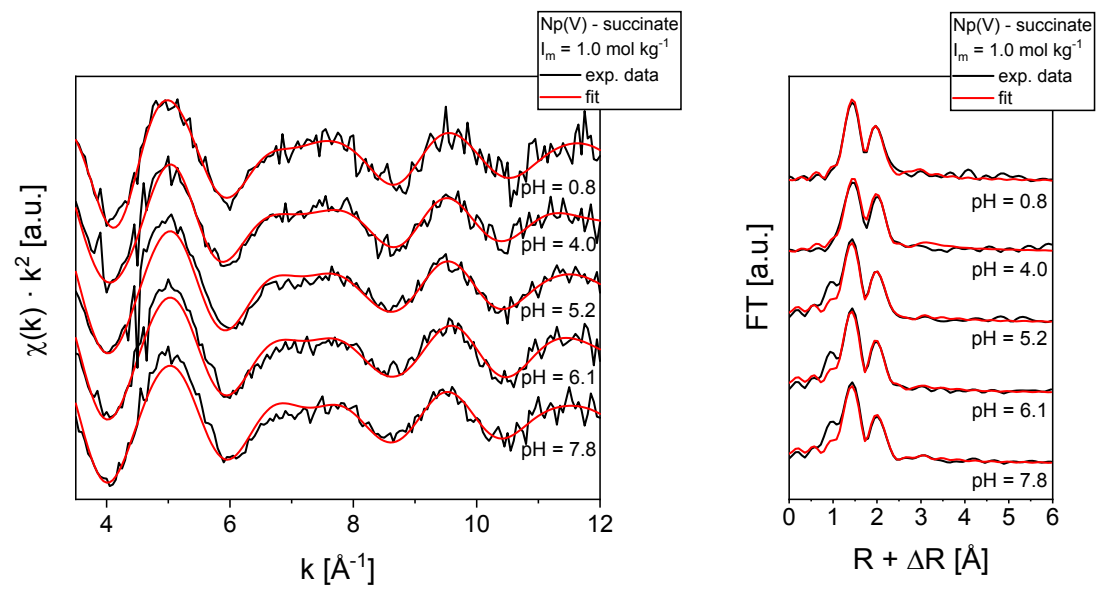

Figure S2: EXAFS analyses of $\mathrm{Np}(\mathrm{V})$ in the presence of succinate as a function of the conditional $\mathrm{pH}_{\mathrm{c}}$ value. (left) raw $\mathrm{k}^{2}$ weighted Np-L $\mathrm{L}_{3}$-edge EXAFS spectra; (right) related Fourier transforms; (black) experimental data; (red) best fit from EXAFSPAK. 